\section{Management of Maritime Pollutants in Shipping and Commercial European Ports Based on Relevant Physical and Biogeochemical Environmental Parameters}

A workshop was held at the Chemical Engineering Dept., Universitat Politècnica de Catalunya, Barcelona, Spain, 4-5 February 2013.

Prof. F. Sakellariadou welcomed the task group members and expressed her thanks to them for participating at the workshop, especially to the team of the Universitat Politècnica de Catalunya for hosting the workshop and making the arrangements. She referred to Dr Simon Bray from the Centre for Environmental Sciences, University of Southampton, who was unable to attend the workshop.

Prof. F. Sakellariadou gave a brief description of the project, emphasizing the overall concept, the methodological approach, the final outcome, and the dissemination plan.

Prof. V.S. Tselentis gave his presentation entitled "Physicochemical parameter monitoring in Piraeus port." He referred to the monitoring procedure applied for several years in Piraeus port, showing sampling sites and describing physicochemical parameters measured in both the water column and the sediment layer. He presented the results of a 16-year ongoing project.

Prof. C. Wooldridge and Marti Puig Duran gave their presentation entitled "Management tools based on sound scientific data." They referred to the seaport area characteristics, emphasizing the growing culture of monitoring and reporting and showing the evolution (with time) of environmental priorities. They noticed that from 1960 to 2010, there was an increase in proactive environmental management systems and environmental awareness. They proposed lists of environmental condition indicators and consolidated environmental management indicators. They presented the stakeholders assessment results of the European Sea Ports Organization Sustainable Development Committee, showing the acceptance versus feasibility of selected environment indicators. They referred to the environmental management index and the sustainability index.

The Task Group Chair and Members had a successful and interesting meeting with Barcelona port authorities. The port manager discussed the environmental monitoring procedure applied in Barcelona port. There was an exchange of ideas regarding environmental port issues.
Prof. R.M. Darbra Roman presented the PERSEUS project, dealing with the assessment of the environmental performance of Mediterranean and Black Sea ports through the use of environmental indicators. PERSEUS refers to a policy-oriented marine environmental research in the southern European seas, including two seas, 53 partners and 21 countries. Objectives and structure of the project were described. Main sections of an environmental questionnaire were reported, with detailed information concerning port profile, activities, environmental aspects, environmental management, environmental performance indicators, and reasons for not implementing Environmental performance indicators (EPIs).

Prof. V.S. Tselentis gave a presentation entitled "Port bio-monitoring for EMS - Piraeus port." He reported detailed information on biota studies on sea water and sediment matrix from the Piraeus port. His presentation included the most probable number (MPN) values for Coliform, E. coli, and Enterococci during the period 2000-2009.

Prof. F. Sakellariadou gave a presentation entitled "Pollutant loads in Lavrio and Elefsina ports." She referred to the characteristics of the ports. She showed total metal classification on the basis of agglomerative hierarchical clustering. She presented sediment downcore fluctuations of heavy metals in geochemical fractions. She emphasized the significance that coastal mineralization and anthropogenic pollution sources have on the marine environmental status. She pointed out the role of marine sediment as an environmental archive for natural and man-made influence on environmental status.

The Task Group, inspired by the hosting country, decided to give the acronym PICASSO to the current project, standing for Performance Indicators of Chemical and Selected Scientific Origin.

For more information, contact Task group Chair Fani L. Sakellariadou $<$ <sakelar@unipi.gr> www.iupac.org/project/2010-028-3-600

\section{Medicinal Chemistry India}

The Medicinal Chemistry India project, a pilot study aimed at improving the educational aspects of medicinal chemistry as applied to drug discovery, held at Sri Ramachandra University, Chennai, India during 11-15 February.2013, was a resounding success. A total of 112 students from industry and academia attended this short course. Industry and academic experts from the US participated in the interactive teaching sessions and discussions. The key organizing and supporting 